\title{
App Review Series: RadioGraphics
}

\author{
Andreas M. Rauschecker ${ }^{1} \cdot$ Tessa S. Cook $^{1}$ \\ Published online: 2 February 2016 \\ (C) Society for Imaging Informatics in Medicine 2016
}

\section{Full Review}

\section{Introduction}

As every radiologist knows, RadioGraphics is one of the premier review article journals in our field. Its educational

App Specs App Name: RadioGraphics, Radiological Society of North America

App Icon URL: https://itunes.apple.com/us/app/radiographicsradiological/id741063884

App Developer: RSNA

App Developer Website: rsna.org

App Price: Free (access to articles with RSNA or institutional membership) Apple App Store URL: https://itunes.apple.com/us/app/radiographicsradiological/id741063884

Google Play Store URL: https://play.google.com/store/apps/details?id=com. sheridan.rsna.radiographics

Category: journal, educational

Tags: educational, free, radiology, journal, iPad-compatible

Works Offline: $Y$

FDA Approval: NA

Quick Review (1 star, lowest; 5 stars, highest)

Overall Rating (1-5): 5

Content (1-5): 5

Usability (1-5): 4.5

Pros: Outstanding and convenient access to all RadioGraphics content, with an overall beautiful interface that functions very well. Can "bookmark" articles of interest.

Cons: Occasionally the user interface is slightly unwieldy. Accessing older content may be cumbersome. Accessing CME content from a smartphone is not much improved by the app compared to going through the web.

At a glance: If you like perusing issues of RadioGraphics to stay up to date, and you do not mind reading on your tablet or phone, then this app is for you. A great interface allows you to review all the images and text from recent issues and bookmark articles in an easy manner.

Andreas M. Rauschecker

andreas.rauschecker@gmail.com

1 Radiology Department, Hospital of the University of Pennsylvania, 3400 Spruce St, Philadelphia, PA 19104, USA

content is an outstanding resource for in-depth topics and matching illustrations for a myriad of topics in radiology. This app succeeds in making all of that terrific content available on your phone or tablet at the touch of a button. Now, you can have all of these illustrations and expert content available at your fingertips.

\section{Features/Content}

As an RSNA member, or with an individual or institutional subscription, one has access to full-text articles and high-resolution images published in RadioGraphics. Without a subscription, access to abstracts is nevertheless free of charge. To make things easy, one needs only to sign in a single time to access all the content; the login information is saved for the next time the app is used. The app developers have incorporated many very useful features, including bookmarking of articles (which unfortunately does not sync across devices), a good search tool, sharing links to article through social media or SMS, sharing images via email, and linking to SA-CME tests. For users that prefer to read their articles within another app, print the article, or save a PDF for any other reason, this is easily accomplished as well.

Browsing or skimming a particular issue's articles or table of contents is very straightforward (Fig. 1)-possibly easier than through the website or in print. The within-article search tool also works very well (Fig. 2). Text and images within the articles are brought up in an ergonomic layout. Furthermore, RadioGraphics' practical "teaching points" are automatically highlighted in red (Fig. 3), making it easy to quickly skim an article.

A critical component of the content in RadioGraphics is the images, and these are adeptly handled within the app. If the user touches an image within an article, a high-resolution version of the image is brought up along with the ability to swipe through all the images in the 


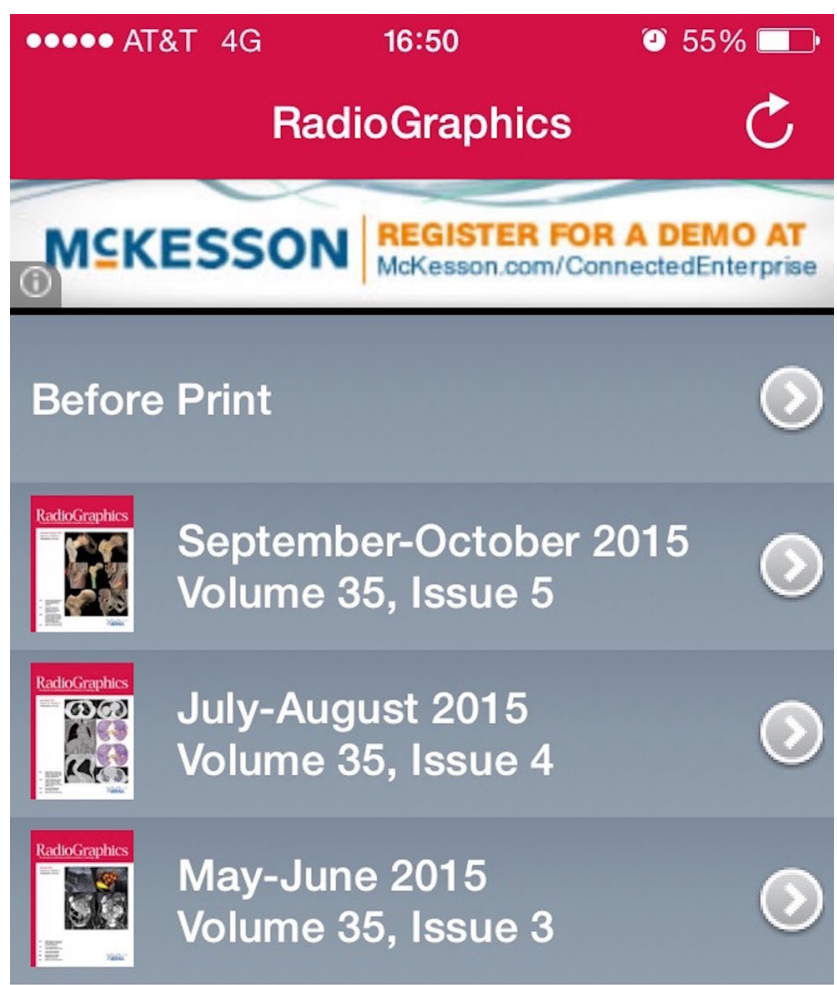

Get More Issues from Server...

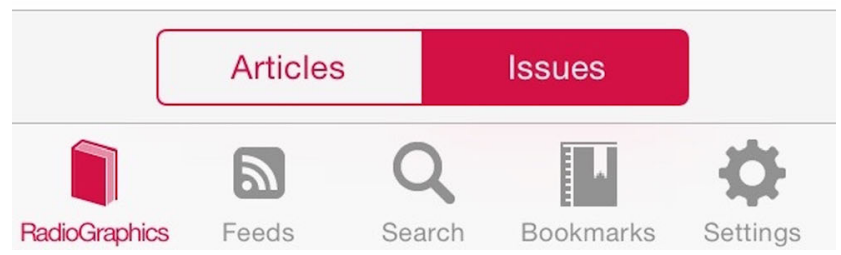

Fig. 1 Table of contents for browsing the latest issues

article (Fig. 4). From here, one can easily save an image to the device or email it to oneself or other interested parties. One minor grievance with emailing is that although the reference for the article is sent along with a thumbnail image and a link to the article, it does not send a high-resolution version of the image - and the figure legend is not included in the email either. Perhaps, there are copyright restrictions limiting what can be done to improve this issue.

If you are worried about all these high-resolution images using too much valuable space on your device, the app developers have thought of this already. The user can

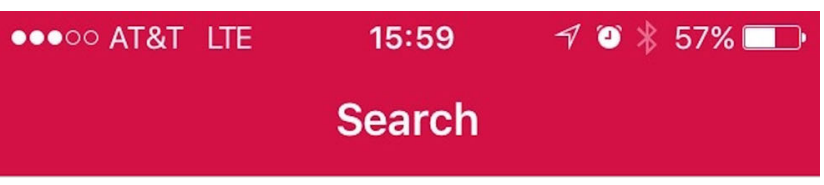

\section{All Content Search}

Search within title, authors, abstract, article body, captions or references...

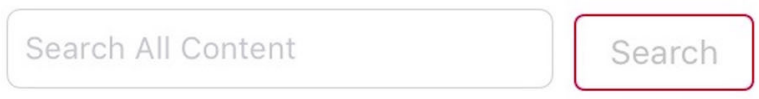

\begin{tabular}{|l|l|l|}
\hline Any Terms & All Terms & Phrase \\
\hline
\end{tabular}

\section{Advanced Search}

Search by Author, Title or Author and Title

\section{Search Authors}

Search Titles

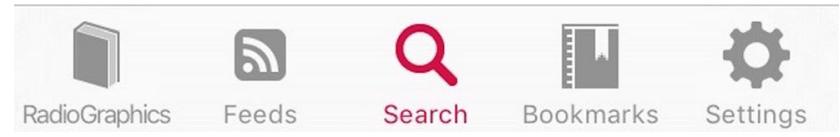

Fig. 2 The search feature primarily searches content downloaded to the device, although an option for searching older and/or archived content does also exist

choose how many days to retain downloaded content, and more importantly, what level of downloaded content detail is cached, ranging from text only to full-resolution images and tables.

\section{Usability}

The app has an agreeable layout and is generally very usable. As mentioned above, articles can be accessed easily by browsing or by searching. The caveat to 


\section{$\bullet \bullet \bullet 00$ AT\&T LTE \\ $15: 53$ \\ 4 ฮ $\$ 57 \%$ \\ Medical 3D Printing for the R...}

We conceptually divide the process into

three parts: image acquisition, image

postprocessing, and 3D printing.

\section{Image Acquisition}

Teaching Point In theory, a 3D medical model can be printed from any volumetric image dataset that has sufficient contrast to differentiate tissues. The imaging data may also be a fusion of images from different (even nonmedical) imaging modalities (Fig 2) (6-8). CT images are most commonly used for 3D printing because of the wide spectrum of applications and relative ease of image postprocessing.

The high contrast, signal-to-noise ratio, and spatial resolution enhance structure differentiation and minimize partial volume effects that could limit 3D printing. Image sections should be reconstructed with isotropic voxels of $1.25 \mathrm{~mm}$ or less (9). Thicker sections compromise model accuracy, while very thin sections (eg, $<0.25 \mathrm{~mm}$ ) require extensive segmentation and STL refinement (see the section on "Image Postprocessing"), particularly in the

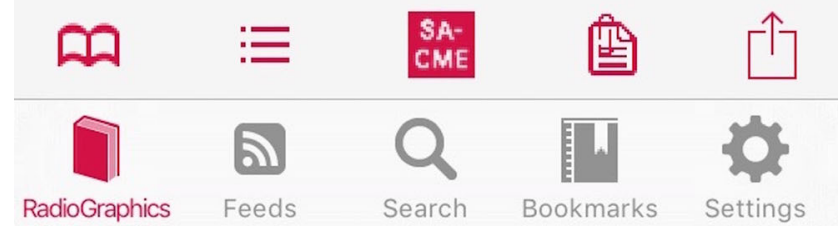

Fig. 3 Teaching points are highlighted in the stylish layout that the app provides. Source, used with permission: [1]

this is that while a search is possible for older RadioGraphics content, searching through the archives for an article older than a few months becomes somewhat clunky, as it requires navigating outside of the app (at the time this review is written).

There are many details included by the app developers that enrich the user experience. For example, references within the article can easily be accessed through hyperlinks (Fig. 5); for users that are on an institutional network, this means being able to jump directly to the primary data when needed.
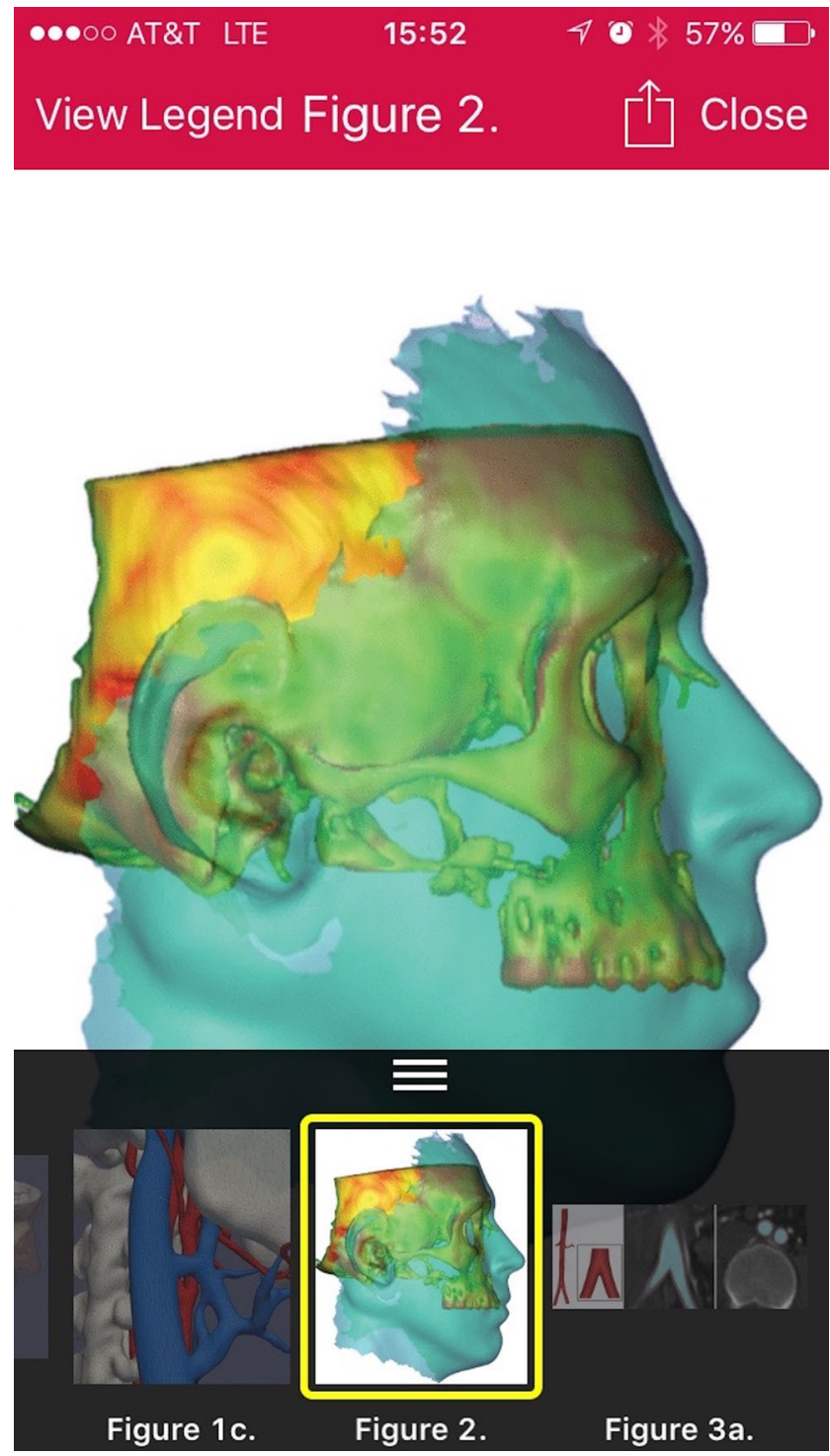

Fig. 4 High-resolution images within an article can be viewed by touching from within the article. One can then swipe through the images or find the images of interest through the thumbnails in the bottom image bar, which is collapsible. Source, used with permission: Mitsouras D, Liacouras P, Imanzadeh A, et al. Medical 3D printing for the radiologist. RadioGraphics 2015;35:1965-1988

Figures can be difficult to handle on tablets or smartphones, but this app does that quite well. For the most part, pulling up images is intuitive, and gestures can be used to zoom, pan, or swipe through images. Images are also embedded beautifully into the text, with collapsible figure legends, and with multi-part figures split up into their parts, thereby increasing readability even on a phone. One oversight here is that the entire 


\section{$\bullet \bullet \bullet \circ \circ$ AT\&T LTE $15: 53$ $40 * 57 \%$ Medical 3D Printing for the R...}

growing) and manual. Although thresholding

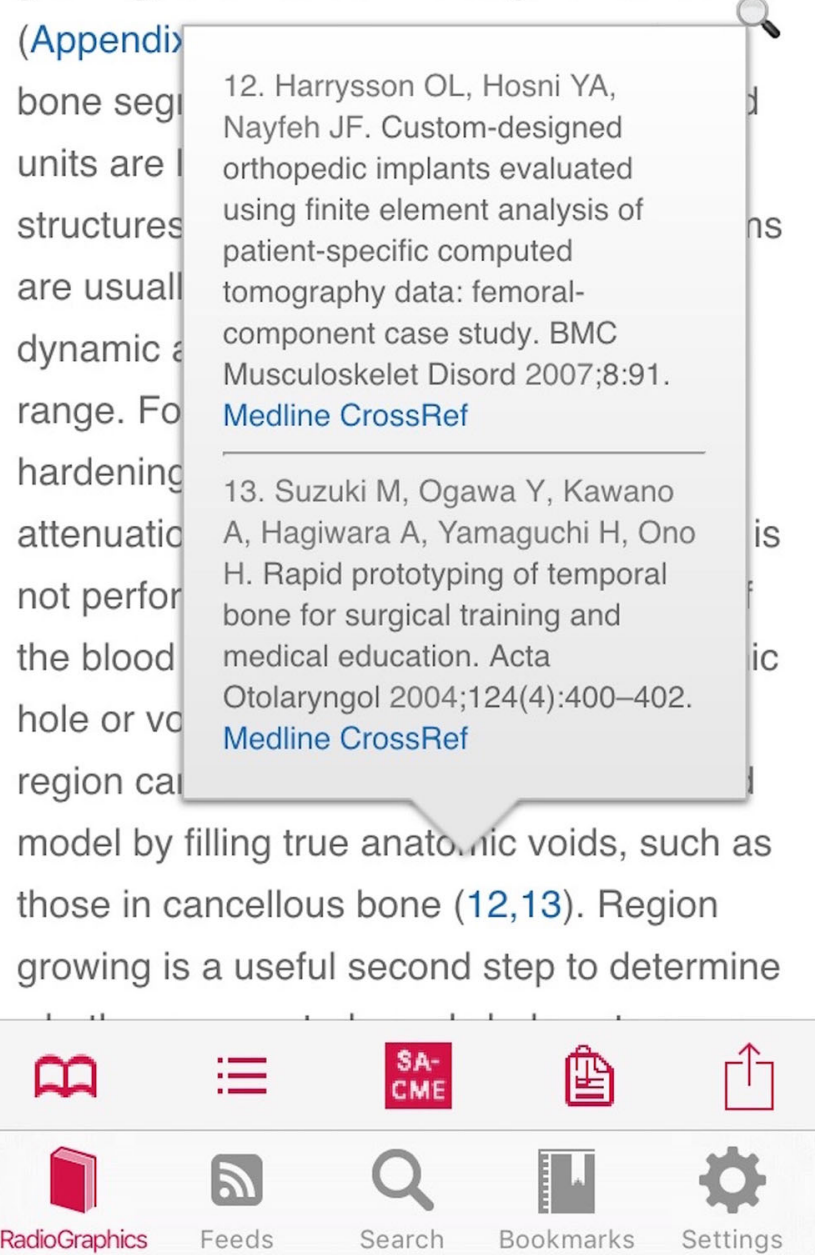

Fig. 5 For those interested, references are very easily accessible through hyperlinks embedded within the content of the app. Source, used with permission [1]

figure legend shows up for each portion of a multi-part figure. It would appear much more streamlined if only the relevant aspect of the figure legend would be shown for each part of the figure.

The CME link is perhaps the portion that needs the most work for usability. Although the direct link is certainly useful, it opens a website within the device's browser, which then requires logging into one's RSNA account again. Having the CME content directly embedded in the app would be a major improvement.

\section{Good}

The RadioGraphics app delivers the finest content in radiology education, as one has come to expect of this premier journal. The developers have obviously taken care in thinking about useful and important features from a radiologist's point of view-for example, quick access to images, bookmarking articles of interest, and sharing of educational content through email. The images show up elegantly on a tablet or a smartphone, and much effort on the backend design has ensured that high-resolution images show up as quickly as possible given the space constraints of many devices.

As discussed above, both text and images are generally managed very well within the app in a userfriendly layout that is particularly well suited to browsing the latest RadioGraphics issues. If one is looking to read an article within the latest issue, the app really shines in landscape mode on the iPad (Fig. 6).

The fact that login information is saved is an immense time-saving measure that will likely convince many radiologists to use the app on a regular basis. It allows easy access to this fine content from anywhere at any time.

\section{Room for Improvement}

There are a few obvious but relatively minor oversights mentioned above that likely will be sorted out in future version of the app, such as being able to email highresolution images and accessing CME directly through the app. In addition, features of the app are occasionally hidden or appear not to work properly unless you know the meaning of certain icons, and this takes a little adjustment. (For example, the image bar at the bottom of the screen appears to have a "slider" for collapsing [see Fig. 4], but it actually requires a simple touch rather than a slide, which is not entirely intuitive.) Here is a tip for another critical feature for anyone hoping to read an entire article on the phone or tablet: to collapse the toolbars on the screen so that only the article itself appears, double tap on the text of the article. Double tap again to make the menu items re-appear. This feature holds true on the phone or tablet.

Accessing the RadioGraphics archives for older articles is currently quite cumbersome from within the app 
iPad ₹

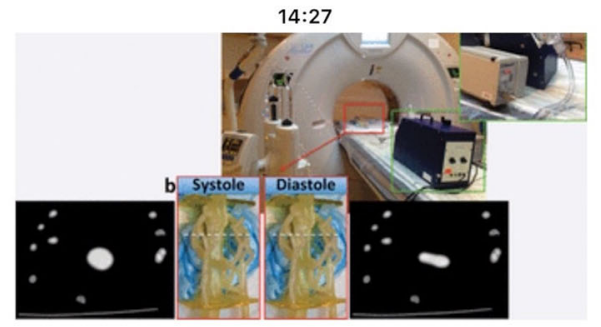

* $96 \%$

Current research (71) to optimize printed vascular model compliance will enhance insight gained from in vitro flow experiments (113), while concurrent in silico (computer-simulated) computational fluid dynamics experiments using STL models from patient data will yield substantial new knowledge and help optimize in vivo imaging techniques such as flow-encoded MR imaging (73), coronary contrast opacification gradients (114), and respiratory dynamics using ${ }^{3} \mathrm{He}$ MR imaging (63). Interventions can similarly benefit; 3D printing of the airways in the nasal cavity has been used to estimate in vitro the differences in air velocity between inspiration and expiration, as well as intercycle vortices toward enhancing nasal operations (115). In the cardiovascular arena, 3D-printed models have been used toward understanding and optimizing the use of stents in difficult coronary bifurcation lesions (116).

\section{Conclusion}

Successful 3D printing from radiologic images is multidisciplinary; accurate models that represent patient anatomy and pathologic processes require close interaction between radiologists and referring physicians.

Teaching Point In addition to implant fabrication, the role of 3D-printed models from DICOM images continues to expand and is fueled by the growing realization that intraoperative utilization of 3D images is not as efficient as having a physical model identical to patient structures, particularly for highly complex interventions. Further reductions in morbidity, mortality, and operating room time $(36,37)$ are inevitable.

However, further, organized, prospective data supporting improved outcomes with use of 3D printing are instrumental for development of guidelines and, ultimately, for reimbursement.

To date, much of our knowledge of 3D printing has been shared among small circles of experts, and publications largely focus on individual cases, with variable reporting. We propose that a format be adopted to enhance communication regarding the reporting of 3D-printed models. This would include all of the following data, if available: printer type, materials, time required to print (assuming the object was printed by itself), estimated cost of materials, and potential overall cost to fabricate the model. The reporting should also include details regarding print layer thickness, modality of the source images (eg, CT), and DICOM section thickness.

Armed with these data for a growing literature, radiologists can begin to amass knowledge, experience, and insights to make 3D printing a reality to better serve patients whose care will benefit from handheld models.

Fig. 6 Landscape mode on a tablet provides a gorgeous reading experience. Source, used with permission: [1]

and is only possible through the advanced search feature, which requires many extra steps, navigates you to the pubs.rsna.org website, and in our hands did not work particularly well—downloads sometimes stalled and downloaded content does not appear within the app but rather within a separate linked website that is difficult to find again at a later time. Conversely, if one has downloaded an issue or article that is no longer needed, it would be helpful to be able to delete it off the device but that is not a feature that is currently supported. Finally, another useful feature that is likely easy to incorporate is the syncing of bookmarks across devices.

Given the host of other features that are managed so well, it would not be surprising to see these features and, likely many others, incorporated into future versions of the app.

\section{Reference}

1. Mitsouras D, Liacouras $\mathrm{P}$, Imanzadeh A, et al: Medical 3D printing for the radiologist. RadioGraphics 35:1965-1988, 2015

Please note that all RadioGraphics content shown here is used with permission by the Radiological Society of North America. 